\title{
Algunas consideraciones sobre historiografía cubana
}

\section{Javier Rodríguez Piña}

El presente trabajo es, de alguna manera, un producto de la investigación que sobre el siglo XIX cubano se realiza dentro del proyecto "América Latina y el Caribe" en el Instituto Dr. José Ma. Luis Mora, y surgió de la necesidad de ordenar la gran cantidad de obras históricas localizadas en México y de la revisión de las bibliografías a nuestro alcance, lo que más tarde nos presionaria a conocer con mayor amplitud la historiografía general de ese país.

Por ello, aunque este artículo no pretende ser exhaustivo, busca por lo menos hacer una clasificación de lo más general, de acuerdo con los distintos periodos en que, para efectos historiográficos, hemos dividido la historia cubana; a saber:

1. De la crónica del descubrimiento a los primeros historiadores cubanos (1492-1750 ca.).

2. De los historiadores primitivos hasta mediados del siglo XIX.

3. Los historiadores del debate nacional (segunda mitad del siglo XlX).

4. Los historiadores de la República.

5. Los historiadores de la Revolución.

Si bien la justificación de los cortes historiográficos se hará sobre el trabajo mismo, es necesario puntualizar que dentro de estas etapas se localizan diversas tendencias y corrientes que muchas veces trascienden y se sobreponen en los distintos periodos. Igualmente, cabe señalar que los tres primeros periodos comprenderán lo que generalmente podriamos estimar como historiografía de la Colonia, que sin embargo se divide para mayor claridad.

No se pretende tampoco hacer un recuento de todo el abundante material que conforma la historiografía cubana, sino evidentemente dar cuenta sólo de las obras que han sido consideradas como las más importantes y cuya información es fundamental para la reconstrucción histórica. Para ello se han revisado los principales análisis historiográficos realizados en el siglo XIX por Felipe Poey y Aloy, ${ }^{1}$ José Antonio Echeverría, ${ }^{2}$ Antonio Bachiller y Morales, ${ }^{3}$ Néstor Ponce de León, ${ }^{4}$ Aurelio Mitjans ${ }^{5}$ y Manuel de la Cruz; ${ }^{6}$ en el actual siglo, por Manuel

\footnotetext{
1 Felipe Poey y Aloy, "Algunos historiadores de la isla de Cuba" en Revista de Cuba, La Habana, marzo de 1880, t. VII, p. 201-209.

2 José Antonio Echeverría, "Historiadores de Cuba" en Revista de Cuba, La Habana, abril 1880, t. VII, p. 381-389 (artículo escrito en 1838).

3 Antonio Bachiller y Morales, Apuntes para la historia de las letras y de la instrucción pública en la Isla de Cuba, La Habana, 1860, t. 2.

" Néstor Ponce de León, "Historia de la Isla de Cuba" en Revista de la Biblioteca Nacional, La Habana, 1911, año III, t. IV, núm. 1-6, p. 97-106 (publicado originalmente en 1888).

5 Aurelio Mitjans, Estudio sobre el Movimiento Cientifico y Literario de Cuba, Habana, Imprenta de A. Alvarez y Compañia, 1890.

- Manuel de la Cruz, "Reseña histórica del movimiento literario en la Isla de Cuba" en Obras de Manuel de la Cruz, Madrid, S. Calleja, 1924-1926, t. I (publicado originalmente en 1891).
} 
Sanguily, ${ }^{7}$ Rafael Montoro, ${ }^{8}$ José María Chacón y Calvo, ${ }^{9}$ Salvador Salazar y Roig, ${ }^{10}$ José A. Fernández de Castro, ${ }^{11}$ Juan J. Remos ${ }^{12}$ y especialmente la síntesis realizada por José Manuel Pérez Cabrera. ${ }^{13}$ Para la historiografía del periodo revolucionario fueron consultados trabajos de Julio Le Riverend, ${ }^{14}$ Manuel Moreno Franginals, ${ }^{15}$ Aleida Plasencia, ${ }^{16}$ el checoslovaco Bohumil Bad'ura ${ }^{17}$ y Jorge Ibarra. ${ }^{18}$

De igual manera fueron de mucha utilidad las entrevistas realizadas en la ciudad de La Habana a los historiadores Julio Le Riverend, Oscar Zaneti, ${ }^{19}$. Francisco Pérez Guzmán y Fé Iglesias, que versaron sobre cuestiones historiográficas, y las conversaciones sobre lo mismo con Enrique López y Sonnia Moro a quienes debo un agradecimiento especial por la ayuda y orientación que me brindaron.

Cuando en 1826 el barón de.Humboldt publica en Paris por primera vez el Ensayo Político sobre la Isla de Cuba, que daría presencia mundial a la isla, comenzaba resaltando la trascendencia que había alcanzado ya a principios del siglo XIX.

"La importancia política de la Isla de Cuba no consiste únicamente en la extensión de su superficie, aunque es doble mayor que la de Haití, ni en la admirable fertilidad de su suelo, ni en sus establecimientos de marina militar y la naturaleza de una población compuesta de tres quintas partes de hombres libres, sino que aún es más considerable por las ventajas que ofrece la posición geográfica de la Habana."'20

7 Manuel Sanguily, Páginas de la Historia, Habana, A. Dorrbecker, 1929, t. I, p. 51-88.

8 Rafael Montoro, "Historiadores de Cuba" en Obra de Rafael Montoro, La Habana, Cultural, 1930, t. 2.

- José María Chacón y Calvo, Cedulario cubano. Los orígenes de la Colonización, Madrid, 1929, p. VII-XVIII.

10 Salvador Salazar y Roig, "La Historia en Cuba" en Anales de la Academia de Histo,ria, La Habana, 1934, t. XIV, enero-diciembre 1932, p. 47-51.

"José A. Fernández de Castro, "Sobre historiadores de Cuba" en Barraca de Feria (18 ensayos y un estreno), La Habana, 1933, p. 136-161.

12 Juan J. Remos, "Historiadores de Cuba" en Revista de la Biblioteca Nacional, año VI, núm. 1, enero-marzo 1955 , p. 45-93.

13 Dos son las obras de Pérez Cabrera que profundizan sobre la historiografía cubana de manera importante. La primera, Fundamentos de la Historia de la Historiografia Cubana, La Habana, Academia de Historia de Cuba, 1959, es un resumen preciso sobre intentos de intelectuales anteriores a él por desarrollar historiografías (la mayoría de autores mencionados en las citas precedentes); la segunda es un trabajo más amplio que cubre sólo hasta el siglo XIX, por desgracia: Historiografía de Cuba, México, Instituto Panamericano de Geografia e Historia, 1962. Ambos fueron de primordial ayuda para el desarrollo de este trabajo.

14 Julio Le Riverend, “Acerca de la historia económica de Cuba: Fuentes y orientaciones" en Ciencias Sociales Contemporáneas, La Habana, núm. 2, diciembre 1965, p. 23-50; igualmente: "De la historia provincial y local de sus relaciones con la historia general de Cuba" en Santiago, Santiago de Cuba, junio de 1982, núm. 461, p. 121-136.

is Manuel Moreno Franginals, "La historia como arma" en Casa de las Américas, La Habana, núm. 40, 1966. p. 20-28.

16 Aleida Plasencia, "Panorama de la historiografia cubana de 1959 a 1967 " en Revista de la Universidad de La Habana, núm. 186-188, julio-diciembre de 1967, p. 91-97.

17 Bohumil Bad'ura, "La historia de Cuba durante el primer decenio socialista" en $R e$ vista de la Biblioteca Nacional "José Marti"', La Habana, septiembre-diciembre, 1970, p. 5-34.

18 Jorge Ibarra, “Algunos problemas teóricos y metodológicos de la historiografía cubana” en Aproximaciones a Clio. La Habana, Editorial de Ciencias Sociales, 1979, p. 293-302.

19 La transcripción de la entrevista con Oscar Zaneti se publica en este mismo número de Secuencia.

20 Alexander von Humboldt, Ensayo Política sobre la Isla de Cuba, París, Casa de Jules Renouard, 1827, p. 1-3. 
"De todas las posesiones españolas, ella es la que más ha prosperado" diría páginas más adelante, ${ }^{21}$ avalando un hecho objetivo que se acrecentaría conforme avanzaba el siglo XIX. La prosperidad de la isla, derivada de todos los factores que señalaba Humboldt, serán el campo de cultivo para el inicio de la historiografía que podemos considerar nacional y que surge evidentemente aparejada con las transformaciones económicas y sociales que ocurren desde finales del siglo XVIII y principios del XIX; y a pesar de que ya se contaba con obras importantes anteriores a dicho periodo, se puede considerar este espacio cronológico como el que pone los cimientos de la construcción historiográfica cubana.

En un primer acercamiento del investigador no cubano a la evaluación cuantitativa inicial que se debe hacer sobre lo que se ha escrito en la isla, es posible afirmar que existe un material abundantisimo que, sin tomar en cuenta por ahora su calidad, coloca a esta nación en un lugar destacado dentro de la producción historiográfica latinoamericana.

Tomas F. Robaina, en el prólogo a la obra Bibliografía de Bibliografias Cubanas, describe la existencia de gran profusión de obras escritas sobre Cuba, basándose en la información del más importante bibliógrafo nacional, Carlos M. Trelles:

"Si comparamos el medio político socio-cultural de Cuba con el existente en aquella época en otras naciones americanas, vemos con cierto asombro, que pocos países podían mostrar una obra de esa magnitud."

"En su 'Bibliografia Cubana' [Carlos M. Trelles] registra un total de 32400 obras, folletos y libros, desglosados por siglos del siguiente modo:

\section{De los siglos XVII y XVIII 1200 \\ Del siglo XIX 23000 \\ Del siglo XX hasta $1916 \quad 8200^{\prime \prime 22}$}

Más de la mitad, o sea 17000 títulos, clasificaría Carlos M. Trelles más adelante, en 1927, dentro de la Biblioteca Histórica Cubana.

Por lo tanto, la necesidad de ordenarla, clasificarla y periodizarla es tarea harto difícil. Se ha optado en principio por la periodización tal como se ha referido al principio, y por incluir dentro de estos cortes a los autores cuya obra se ha desarrollado y publicado en esos límites; es decir, no se hace una clasificación temática en general que incluya a todos los autores que han trabajado sobre un tema o sobre un periodo. Al mismo tiempo se sitúan algunas de las principales corrientes - cuando las hubo - dentro de un mismo periodo, las que se relacionan con la situación política, económica y social de la isla en un momento determinado.

21 Ibidem, p. 6.

22 Tomás F. Robaina (comp. y prólogo), Bibliografía de Bibliografias Cubanas, La Habana, Biblioteca Nacional José Martí, 1973, p. 21. 


\section{De la crónica del descubrimiento a los primeros historiadores cubanos (1492-1750 ca.)}

Cristóbal Colón descubre América en 1492 y en el mismo año pisa lo que vendría a ser el territorio cubano. Pero Colón no sólo se convertirá en el descubridor de la isla de Cuba, también será el primero en dar cuenta al mundo occidental de su existencia.

Así los descubridores, y más tarde los conquistadores, serán los encargados de iniciar la historiografía cubana que, al igual que en el resto de América, se plasmará en crónicas, relatos, cartas, documentos, informes y alusiones hechas en la mayoria de los casos, no con el fin determinado de dejar constancia histórica de la existencia de la isla, sino fundamentalmente con el fin práctico de describir o informar sobre algún hecho particular de la conquista y la colonización.

Característica de esta primera etapa de la historiografía en los cronistas fue la de referirse a la isla de Cuba a veces sólo marginalmente, sin dedicar mayor atención que la que requeria el dar noticia de la presencia española en ella; no pueden considerarse por tanto obras históricas por sí mismas sino como fuentes de las que abrevaron los historiadores posteriores.

Entre los cronistas que en sus obras se ocuparon de la isla de Cuba de manera importante están el descubridor y "primer historiador" Cristóbal Colón quien consignaría en sus diarios la existencia de este territorio; ${ }^{23}$ Pedro Mártir de Anglería, enviado por los reyes católicos para reportar día a día el descubrimiento de los nuevos territorios; ${ }^{24}$ el hijo del descubridor, Hernando Colón, quien escribió una historia de vida de su padre donde transcribe fragmentos de los viajes que éste realizó; ${ }^{25} \mathrm{el}$ primer cronista oficial de las indias nombrado en 1632, Gonzalo Fernández de Oviedo, quien dedica parte de su obra a la descripción de la isla y la historia de su conquista; ${ }^{26}$ la versión del padre Bartolomé de las Casas que ha sido considerada como la más acabada y documentada crónica de la conquista de $\mathrm{Cuba}^{27}$ y la del cronista mayor de Indias, Antonio de Herrera, quien, utilizando el trabajo de sus predecesores, logra escribir una crónica de lo más coherente. ${ }^{28}$

Existe también un grupo de cronistas que sólo se refieren ocasionalmente a Cuba, entre los que cabría mencionar a López de Gómara, ${ }^{29}$

23 El Diario del primer viaje de Colón, hasta donde se sabe, se encuentra extraviado y sólo se conoce por el extracto que de él hace el padre Las Casas en su Historia de las Indias. Reproducido también en: Cristóbal Colón, Diario del Primer Viaje, La Habana, Publicaciones del Instituto de la Víbora.

${ }_{24}$ Pedro Mártir de Angleria, colección de cartas reunidas en el Opus Epistolarum y Décadas de Orbe Novo, publicadas traducidas del latín, México, José Porrúa e hijos, 1964-65, 2 vols.

${ }^{25}$ Hernando Colón, Historia del Almirante Don Cristóbal Colón, México, Latino Americana, 1958.

${ }^{26}$ Gonzalo Fernández de Oviedo, Historia General y Natural de las Indias, Islas y Tie. rra Firme del Mar Océano, Madrid, Atlas, 1959, 5 vols.

${ }^{27}$ Bartolomé de las Casas, Historia de las Indias, México, Fondo de Cultura Económica, 1981, 3 vols. Las Casas tardó 35 años en escribir y recoger información para su obra que terminaría en 1559 y se publicaría en 1875 debido a la polémica que provocó sobre los indigenas americanos.

${ }^{28}$ Antonio de Herrera, Historia general de los hechos de los castellanos en las islas y la tierra firme del mar océano, Asunción de Paraguay, Guaranía, 1944.

29 Francisco López de Gómara, Segunda parte de la crónica general de las Indias, que trata de la conquista de México, Barcelona, Iberia, 1954, 2 vols. 
Diaz del Castillo, ${ }^{30}$ Torquemada, ${ }^{31}$ Solis, ${ }^{32}$ Garcilaso, ${ }^{33}$ González de Barcia $^{34}$ y González Dávila, ${ }^{35}$ quienes describieron el proceso de conquista y colonización de otras zonas americanas.

Otras fuentes que a la larga se convirtieron en material historiográfico son los relatos de viajeros europeos que desde el siglo XVI llegaron a la isla de Cuba por diferentes motivos. Algunas veces era el deseo de conocer y aventurarse por "el nuevo mundo" y posteriormente narrar lo visto a lectores europeos ávidos de conocer América aunque sólo fuera por lecturas; en otros casos, visitantes cuyo viaje tuvo motivaciones cientificas y recorrieron Cuba y toda la América con objetivos geográficos, naturales y etnográficos, y que se encargarían de difundir en el antiguo continente la faz de América.

Entre los viajeros de diferentes nacionalidades que dejaron constancia interesante de su paso por Cuba en este periodo, encontramos al cosmógrafo español Martín Fernández de Enciso quien, en su obra geográfica, dedicó algunas páginas a la isla de Cuba: ${ }^{36}$ al también espanol Pedro Ordonez de Ceballos que viajo poı muchas partes de América y dedica a Cuba una buena parte de su libro; ${ }^{37}$ el italiano Girolamo Benzoni, viajero durante 14 años por América, que hizo una narración de sus travesías por el continente y por la isla, donde destacan los ataques piratas a La Habana; ${ }^{38}$ el inglés Thomas Gage, quien quiso dar en su libro ${ }^{39}$ una visión de los territorios españoles para que sus connacionales se interesaran en ellos, dedicando dos capítulos a Cuba; el francés César de Rochefort, quien escribe en general sobre las Antillas; ${ }^{40}$ Alex Olivier Exquemelin, holandés, también vendría a América y escribiria sobre ella destinando varios pasajes a Cuba. ${ }^{41}$

Otros franceses se ocuparian de lo mismo: Jean Baptiste Labat, aunque haría su trabajo sobre las posesiones francesas en el Caribe, aporta-

30 Bernal Díaz del Castillo, Historia verdadera de la conquista de la Nueva España, México, Ed. Porrúa, 1977. Impresa por primera vez en 1632.

31 Juan de Torquemada, Monarquía indiana, México, Ed. Porrúa, 1975. Impresa originalmente en 1615 .

${ }^{32}$ Antonio de Solis, Historia de la conquista, población y progresos de la América septentrional, conocida con el nombre de Nueva España, México, Editora Nacional, 1959, 2 vols.

${ }_{33}$ Garcilaso de la Vega, "Ensayo cronológico para la historia general de la Florida", Obras completas, Madrid, Castalia, 1964.

${ }_{34}$ Andrés González de Barcia, Ensayo cronológico para la historia general de la Florida, Madrid, Imprenta Real, 1923.

${ }_{35}$ Gil González Dávila, Teatro eclesiástico de las primitivas iglesias de las Indias Occidentales: vida de sus obispos y cosas memorables de sus sedes, Madrid, Ed. José Porrúa, 1959.

36 Martín Fernández de Enciso, Suma de geografia, Sevilla, Imprenta de Jacobo Cromberger, 1519. La parte de Cuba está reproducida en las Memorias de la Real Sociedad Patriótica de la Habana, La Habana, Oficina del Gobierno y Capitania General, 1837, t. III, p. 357-362.

${ }_{37}$ Pedro Ordóñez de Ceballos, Viaje del Mundo, Maddrid. Luıs Sánchez Impresor Real, 1614.

${ }^{38}$ Girolamo Benzoni, La historia del Mundo Nuovo, Venecia, Imprenta de Franceso Rampazetto, 1565.

${ }_{39}$ Thomas Gage, The English-American, his travail by sea and land, or a new survey of the West Indias, Londres, 1648. La versión española: París, Librería de Rosa, 1838.

${ }^{40}$ César de Rochefort, Histoire naturelle et morale des îles Antilles de l'Amérique, Rotterdam, Chez Arnould Lerrs, 1658.

${ }^{41}$ Alex Olivier Exquemelin, Americaensche Zeerovers, Amsterdam, 1678. Traducido al español con el título de Piratas de la América, y luz a la defensa de las costas de Indias Occidentales, La Habana, UNESCO, 1963. El tema del libro es, como su nombre lo indica, una historia del filibusterismo en América. 
ria datos interesantes sobre la región que aprovecharían escritores posteriores; ${ }^{42}$ y Charlevoix, quien escribe la historia de la isla de Santo Domingo, pero hace útiles referencias acerca de Cuba. ${ }^{43}$

Cabe mencionar también en este primer periodo a otros tres historiadores europeos, Raynal, ${ }^{44}$ Robertson ${ }^{45}$ y Muñoz, ${ }^{46}$ que a pesar de escribir su obra ya en la segunda mitad del siglo XVIII, en pleno auge del enciclopedismo, y sin viajar a América, logran integrar cada uno por su cuenta textos históricos que llegaron a convertirse en clásicos dentro de su género.

\section{De los historiadores primitivos hasta mediados del siglo XIX}

Quizá uno de los elementos que caracteriza con mayor fuerza a la historiografía cubana es su tardia aparición como historiografía autóctona. Hacia la primera mitad del siglo XVIII, dos siglos después de la colonización española, no han aparecido todavia los sujetos capaces de plasmar el pasado y el presente de la isla ni como historia ni, salvo el caso de Silvestre de Balboa, ${ }^{47}$ como literatura.

Esta floración tardia de la literatura y la historia cubana puede ser explicada por varios factores. Por un lado, el pobre desarrollo cultural que hasta el siglo XVIII se debe a la inexistencia de centros de enseñanza en la isla; por el otro, la ausencia de los grandes temas originados en la isla que tal vez hubieren empujado a la formación de cronistas cubanos.

Es necesaria la transformación de la sociedad cubana que se inicia en el siglo XVIII para que aparezcan las primeras manifestaciones vernáculas de historiografía: el embate contra el latifundio ganadero que había determinado la economía cubana durante los siglos XVII y XVIII se lleva adelante positivamente mediante la agricultura comercial. Esta se fue convirtiendo, poco a poco, a lo largo del siglo XVIII, en el eje sobre el cual fue girando la rueda de la historia económica de Cuba.

42 Jean Baptiste Labat, Nouveau voyage aux isles de l'Amérique, París, chez Guillaume Cavelier, 1722; editada en español como: Jean Lavat, Viaje a las islas de la América, La Habana, Casa de las Américas, 1979.

43 Pierre-François-Xavier de Charlevoix, Histoire de l'isle Espagnole ou de S. Domingue, París, Prélard, 1730.

${ }_{44}$ Guillaume-Thomas-François Raynal, filósofo e historiador enciclopedista francés, en su obra Histoire philosophique et politique des établissemens et du commerce des Européens dans les deux Indes, Amsterdam, 1771 (traducida al español como Historia Politica de los Establecimientos ultramarinos de las naciones europeas, Madrid, Antonio de Sancha, 1784), consagra una buena parte a la historia de la isla de Cuba.

${ }^{45}$ William Robertson, escocés, rector de la Universidad de Edimburgo, escribió una narración basada en el material de los cronistas del nacimiento del imperio español en América: History of America, Londres 1777. También traducida al español: Historia de América, Burdeos, Imprenta de Pedro Beame, 1827.

46 Juan Bautista Muñoz fue designado por la Corona española para que escribiese, basándose en documentos, la historia del "nuevo mundo" con el fin de rebatir las interpretaciones de Raynal y Robertson. Tuvo acceso a muchísimas fuentes pero al final su interpretación no contradijo las ideas de los otros autores. Sólo se publicó un volumen pues la muerte le impidió continuar su obra. Historia del Nuevo Mundo, Madrid, Viuda de Ibarra, 1793.

47 Silvestre de Balboa Troya y Quezada escribió el poema histórico Espejo de Paciencia en 1608 , convirtiéndose en "el más antiguo monumento literario de Cuba" del cual se tenga conocimiento, según Pérez Cabrera. Sin embargo y aunque relata un hecho histórico -el secuestro y rescate del obispo fray Juan de las Cabezas - es más bien un ejercicio literario que histórico. Además, Silvestre de Balboa fue originario de la Gran Canaria aunque avecindado en la isla de Cuba. 
El proceso de transformación agrícola y el impulso al comercio se complementaron, en la segunda mitad del siglo XVIII, con la política administrativa que la corona española intenta con objeto de modernizar su relación con América y lograr una más eficaz explotación de su riqueza.

Pero definitivamente, sin dejar de lado el debate que sobre este punto existe en la historiografia cubana, el hecho que vendrá a imponer con mayor fuerza transformaciones económicas para la sociedad cubana será sin duda la política resultante de la ocupación inglesa en la ciudad de La Habana en 1762. No porque tal ocupación hubiera sido la causa de los cambios que ya se estaban dando en el seno de la economía, sino por lo que este hecho significó globalmente, sobre todo para el comercio entre Europa y América y, en el caso cubano, en el impulso a la plantación azucarera.

Para fines del siglo XVIII y principios del XIX, la economía y la sociedad cubana han sufrido ya un proceso irreversible de transformación que inevitablemente estará acompañado por el surgimiento de lo que más adelante se va a considerar como la "cultura nacional", cuyas bases podemos encontrar a lo largo del siglo XVIII pero principalmente en la segunda mitad, entre los siguientes hechos: en 1723 se instala la primera imprenta en la isla: 5 años más tarde se funda la Universidad de San Jerónimo en La Habana; en 1764 se funda el primer periódico cubano, La Gaceta; para 1773 se establece en La Habana el Seminario de San Carlos que sería por algún tiempo, y por los personajes que lo dirigieron, quizás más importante que la Universidad misma; en 1774 se realiza el primer censo en Cuba; en 1790 se funda el primer periódico literario que tendría profunda influencia en la intelectualidad cubana, el Papel Periódico de La Habana; hasta aquí por llegar sólo hasta inicios del siglo XIX.

Lo que es claro es que este campo de cultivo de la historiografía vernácula de la segunda mitad del siglo XVIII y primera del XIX, estará ligado a la definición de la economía en función de la agricultura comercial. Por ello, y sin pretender establecer una correspondencia mecánica, evidentemente la conciencia histórica de intelectuales del momento aparecerá ligada principalmente a los intereses de la oligarquía terrateniente y comercial. ${ }^{48}$

Conocida como la primera obra histórica que aparece en el siglo XVIII y considerada como propiamente cubana, ${ }^{49}$ sin serlo del todo, será la Historia de la isla y catedral de Cuba de Pedro Morell de Santa

${ }^{48}$ Julio Le Riverend conceptúa este proceso en los siguientes términos: "Subrayemos que el gran movimiento de la cultura nacional se inicia a fines del XVIII alzándose rápidamente sobre las escasísimas realizaciones de la cultura tradicional o escolástica. El racionalismo moderno venció finalmente. Además, este impulso de la cultura coincide con un portentoso desarrollo económico que se produce entre 1780 y 1820 , cambiando radicalmente las condiciones sociales concretas del pais. Lógicamente, están en primer plano, desde entonces, ciertas conquistas básicas de la ciencia: estudio de la naturaleza, experimentalismo, mecánica, etc. Toda la actividad cultural está impregnada de un sentido práctico que la relaciona estrechamente con las cuestiones del gran cambio económico y social. A la cabeza de este cambio, se encuentra la clase de los terratenientes fabricantes de azúcar que se esfuerzan por darle a la cultura ese giro práctico". "Acerca de la Historia..." op. cit., p. 24.

${ }^{49}$ Existen referencias de la existencia de un manuscrito inédito y perdido del que se supone primer cubano que se ocupó de la historia cubana, Ambrosio Zayas Bazán, quien en 1725 compuso la Carta y Relación de la isla de Cuba y sus particularidades, con tres historias de los gobernadores de la Habana desde el año de 1549 hasta 1725; de los Obispos hasta 1705 y de los Virreyes en México. 
Cruz, ${ }^{50}$ donde en 1760 describe la historia del descubrimiento, conquista y población de la isla hasta 1752.

Contemporáneo de Morell de Santa Cruz, termina en el mismo año (1760) Joseph de Ribera su obra Descripción de la Isla de Cuba ${ }^{51}$ que sería una ojeada histórica y una descripción de la economía, sociedad, naturaleza y política de Cuba.

Un año más tarde, en 1761, culmina su obra José Félix de Arrate, considerado por la crítica como el último representante de la vieja oligarquia. Su obra Llave del Nuevo Mundo... ${ }^{\text {s2 }}$ se convertiría, sin embargo, después de su publicación en 1830 , en un clásico dentro de la historiografía cubana por su asomo crítico y los elementos de reivindicación nacional, a pesar del escolasticismo ${ }^{53}$ que contiene y de las críticas de Urrutia y del padre Caballero.

La primera obra histórica que se imprime en la isla se debe a la autoría de fray José María de Peñalverde en $1781,{ }^{54}$ pero pierde su mérito ante el hecho de no constituir un trabajo serio sino coyuntural y por encargo de la corona española, aunque sí representativo del periodo.

Otro autor considerado clásico por los historiógrafos, Ignacio José de Urrutia y Montoya, dará á conocer en 1789 el Teatro histórico..., ss un intento más por historiar la dominación española en la isla, quedándose únicamente en el primer tomo de los veinte que se propuso escribir.

Para finales del siglo XVIII otro factor que imprimirá vigor a las profundas transformaciones que ha venido sufriendo la isla de Cuba, será la presencia, como gobernante, del general Luis de las Casas, quien entre 1890 y 1896 impulsará reformas económicas y sociales que serán determinantes para el futuro del país. La fundación de la "Sociedad Económica de Amigos del Pais" en 1793 y del "Real Consulado de Agricultura y Comercio" serán de los hechos que surgirán al amparo de este gobernante y sus colaboradores cubanos, entre quienes destacan Arango y Parreño y el padre José Agustín Caballero principalmente. Ambos personajes cubanos tendrán una importancia fundamental para la definición de lo que vendrá a cuajar como la "cultura cubana", sobre todo por la superación y critica al escolasticismo que hasta en-

so Pedro Agustín Morell de Santa Cruz, natural de Santo Domingo, pasó a vivir a Cuba en 1717 donde llegó a ser obispo. Escribió la Historia de la isla y catedral de Cuba que publicaría por primera vez en 1929 la Academia de Historia de Cuba.

s1 Nicolás Joseph de Ribera, Descripción de la Isla de Cuba, La Habana, Ciencias Sociales, 1975.

52 José Félix de Arrate, Llave del Nuevo Mundo, antemural de las Indias Occidentales. La Habana descripta: noticias de su fundación, aumentos y estado, México, Fondo de Cultura Económica, 1949.

s3 Manuel de la Cruz se refiere en términos hipercríticos a los primeros historiadores: "Los primeros historiadores cubanos reproducen el tipo intelectual del primimitivo cronista: monótonos, difusos, oscuros, escritores menos que mediocres, sus narraciọnes, sin colorido ni arte, son apuntes cronológicos". Op. cit., p. 15. En cambio, más mesurado, Juan J. Remos dirá 30 años más tarde: "La lectura de los primitivos historiadores de Indias inclinó a nuestros primeros historiadores al sentido de crónica que imprimieron a sus obras. Fueron verdaderos cronistas, que tomaron de aquéllos sus preciosos datos y añadieron a éstos cuanto ellos pudieron captar de sucesos y personajes más cercanos, e incluso de sus contemporáneos", op. cit.. p. 46.

54 José María de Peñalverde, Idea Geográfica, histórica y política de la isla de Cuba y ciudad de La Habana que se publicó en el primer Calendario Manual y Guia de forasteros de 1781, en La Habana.

55 Ignacio José de Urrutia y Montoya, Teatro histórico, jurídico y político militar de la Isla Fernandina de Cuba, principalmente de su capital La Habana, La Habana, UNESCO. 1963. 
tonces predominaba en los autores cubanos. ${ }^{56}$

Hay que nombrar a otros dos escritores presentes en los años finales de este mismo siglo: Buenaventura Pascual Ferrer y Tomás Romay. El primer relato de viajes de un cubano ${ }^{57}$ se debe a Pascual Ferrer y Romay, cofundador con el padre Caballero de Papel Periódico y de la Sociedad Económica de Amigos del País; es autor de un estudio sobre las fundaciones pías del país. ${ }^{58}$

Ya en la primera mitad del siglo XIX la historiografía nacional se intensificará con la aportación de numerosos trabajos que van a tener como preocupación fundamental el rescate del pasado unido al estudio de los problemas esenciales de la sociedad cubana.

En 1830 comienza a funcionar, si bien con poca eficacia, la Sección de Historia de la Sociedad Patriótica de La Habana, antigua Sociedad Económica de Amigos del País creada en 1793. Las primeras tímidas expresiones de esta etapa se traducen en el intento de editar algunas de las obras sobre la historia de la isla que se encontraban inéditas, como la de Arrate, que fue la única que se publicó realmente. Pero antes que esto ocurriera, el nuevo siglo procreó obras interesantes, producto de las inteligentes plumas de historiadores cubanos como el popular José Antonio Valdés, ${ }^{59} \mathrm{o}$ el furibundo absolutista José Ma. Calleja ${ }^{60} \mathrm{M}$ as la obra que definitivamente será fundamental en la primera mitad del siglo XIX será El Ensayo Político sobre la Isla de Cuba ${ }^{61}$ del sabio alemán Alejandro de Humboldt.

Siguiendo los pasos de Humboldt y completando con él lo más importante de la historiografía cubana de la primera mitad del siglo XIX - aunque sus obras se continúan hasta la siguiente mitad- están dos prolificos historiadores españoles cuya amplia obra los consagra, independientemente de las concepciones políticas de ambos: Ramón de la Sagra ${ }^{62}$ y Jacobo de la Pezuela. ${ }^{63}$

56 Tanto Francisco Arango y Parreño, como José Agustín Caballero deben considerarse evidentemente como los más importantes pensadores del siglo XVIII, aun cuando la influencia de su pensamiento abarque parte importante del siglo XIX. Representan ambos las ideas modernizantes de los terratenientes que luchan por el libre comercio. En sus obras sobresale una preocupación crítica fundamental más que el interés directo por historiar la nación. Véase: Francisco Arango y Parreño. "Discurso sobre la agricultura de La Habana y medios de fomentarla" en Obras de Francisco Arango y Parreño, La Habana, 1888, t. 1, p. 52-126; José Agustín Caballero, Escritos Varios, 2 t., La Habana, Universidad de La Habana, 1956.

57 Buenaventura Pascual Ferrer, Viaje a la isla de Cuba, Madrid, 1798; publicado en La Habana en 1877 bajo el título "Cuba en 1789" en la Revista de Cuba.

s8 Tomás Romay, "Discurso histórico moral sobre la fundación y progresos del hospital San Francisco de Paula de La Habana" (1799) publicado por primera vez en 1849 en las Memorias de la Sociedad Económica de La Habana, t. XX, p. 489-499.

59 Antonio José Valdéz, Historia de Cuba y en especial de La Habana, La Habana, UNESCO, 1964, impresa originalmente en 1813, sería la primera historia de Cuba publicada.

${ }^{60}$ José Ma. Calleja, Historia de Santiago de Cuba. Compuesta y Redactada en vista de los manuscritos originales inéditos de 1923. Escrita en ese mismo año, pero publicada por primera vez en La Habana, Imprenta "El Universal", 1917.

61 Alejandro von Humboldt, Ensayos Políticos sobre la Isla de Cuba, París, Jules Renouard, 1829; publicada por primera vez en francés en 1826. Humboldt estuvo en Cuba en dos ocasiones, la primera de diciembre de 1800 a marzo de 1801 y la segunda durante abril-mayo de 1804 . Tuvo acceso a todo tipo de información tanto del gobierno como de particulares que colaboraron gustosamente con él, lo cual le permitió escribir una obra que dio reconocimiento mundial a la isla de Cuba.

62 Ramón de la Sagra fue un escritor y naturista gallego que llegó como funcionario a Cuba en 1820 para dirigir el Jardín Botánico de La Habana y comenzó a reunir el material que posteriormente daría origen a vasta obra. Lo más importante es Historia econó- 
Otro europeo cuya obra forma parte de la historiografia cubana de la primera mitad del siglo XIX será David Turnbull, ${ }^{64}$ irlandés abolicionista que siendo cónsul inglés llegó a ser miembro correspondiente de la Sociedad Patriótica de la que fue expulsado por sus ideas "radicales".

\section{La historiografia del debate nacional. Segunda mitad del siglo XIX}

En la segunda mitad de este siglo y sobre todo en lo que va de 1868 a 1898 encontramos lo que se puede caracterizar como el periodo de la lucha por el forjamiento de la nacionalidad cubana. En este lapso se llevan a cabo dos guerras por la independencia de Cuba y por lo tanto es cuando las definiciones políticas de los intelectuales de la época se van a dar con mayor claridad.

Sin homologar los contenidos de la guerra de los diez años (18681878) con la que se inicia en 1895 , es evidente que lo que caracteriza a ambas será, por supuesto, la lucha por lograr el fin del coloniaje español. En general, esta lucha se reflejará en los análisis políticos que se realizan en el periodo, mismos que pasarán a formar parte de la historiografía de la época. De hecho, lo esencial de la historiografía de esta fase estará consituido por el debate entre Cuba y España, y entre independencia, autonomia y colonia.

Por lo tanto podemos marcar una división - arbitraria como todasentre los autores de este tiempo de acuerdo con las tendencias políticas reflejadas en sus obras. Por un lado tenemos a los autores identificados con la independencia, aun cuando no lo expresen directamente; por otro, a aquéllos que proclaman su apego al autonomismo, es decir, la línea reformista en el debate, y por supuesto los que declaran a toda costa su apego a España.

En la lista más larga, la de los independentistas, podemos localizar a Pedro José Guiteras, quien aun cuando no revela en sus obras su inclinación hacia el independentismo, si expone una crítica al coloniaje, tan profunda que lo incluye en esta línea; ${ }^{65}$ a Pedro Santacilia, poeta independentista ${ }^{66}$ que, expulsado de Cuba, vendria a México a luchar al

mica, politica y estadística de la Isla de Cuba, o sea de sus progresos en la población, la agricultura, el comercio y las rentas, La Habana, Imprenta de las Viudas de Arazoza y Spler, 1831; también Historia Fisica, Política y Natural de Cuba, 12 vols., Paris, 1838-1842. Sagra puede considerarse a fin de cuentas, y a pesar de la polémica con José Antonio Saco, un liberal progresista influido por las más avanzadas tesis del enciclopedismo.

63 Jacobo de la Pezuela es, en contraposición a Ramón de la Sagra, la voz oficial de España y el principal apologista del coloniaje español. Sin embargo su obra es imprescindible para la historiografia cubana y quizá de lo más importante hasta ese momento. Recordemos sólo su Ensayo hisórico de la isla de Cuba, Nueva York, Imprenta española de R. Rafael, 1842; en 1863 comenzó la publicación del Diccionario geográfico, estadístico, histórico de la isla de Cuba, Madrid, Imprenta del Establecimiento de Mellado, 18631866; Historia de la isla de Cuba, Madrid, Carlos Bailly-Bailleri, 1868-1878; esta última se considera la mejor obra de Pezuela.

${ }^{64}$ David Turnbull, Travels in the West, Cuba; with notices of Porto Rico, and the Slave Trade, Londres, Longman, 1840.

65 Pedro José Guiteras, considerado por Manuel de la Cruz como el mejor en historiografia cubana (op. cit., p. 24), es quizá el autor con el que da comienzo la visión crítica de la historia cubana, es decir, se comienza a romper la llamada "crónica histórica" por la historia crítica. Entre sus obras: Cuba y su Gobierno, Londres, 1853; Historia de la conquista de La Habana, Filadelfia, Parry and MacMillan, 1856; Historia de la isla de Cuba (publicada originalmente en 1865-66), La Habana, Colección de libros cubanos, 1927.

${ }_{6} 6$ Pedro Santacilia, Lecciones orales sobre la historia de Cüba, pronunciadas en el Ateneo Democrático Cubano de Nueva York, Nueva Orleáns, Imprenta de Luis Eduardo del Cristo, 1859. 
lado de Juárez contra el Imperio; a Enrique Piñeyro ${ }^{67}$, otro de los más importantes historiadores críticos; Juan Arnao $^{68}$ que fue incluso condenado a muerte por sus actividades políticas junto a Narciso López; Néstor Ponce de León quien se propone escribir una nueva versión de la historia de $\mathrm{Cuba}^{69}$ y Manuel Sanguily. ${ }^{70}$

Junto a estos autores aparecen otros que con menores pretensiones historiográficas narran los hechos y luchas; son los cronistas de los movimientos revolucionarios desde el punto de vista de la independencia, y entre ellos podemos señalar a los siguientes, en el orden cronológico de la aparición de sus obras: Francisco Javier Balmaceda, ${ }^{71}$ Francisco Javier Cisneros, ${ }^{72}$ Fermín Valdés Domínguez, ${ }^{73}$ Antonio Zambrano, ${ }^{74}$ Ramón Roa, ${ }^{75}$ Manuel de la Cruz, ${ }^{76}$ Enrique Collazo, ${ }^{77}$ Nicolás Heredia, ${ }^{78}$ Rafael Maria Merchán, ${ }^{79}$ Fernando Figueredo. ${ }^{80}$.

Mención aparte merece la figura de Antonio Bachiller y Morales,

${ }^{67}$ La biografia y la monografia histórica serán las formas preferidas de Piñeyro, a lo que hay que añadir también su actividad revolucionaria. De entre su amplia obra sobresalen Morales Lemus y la Revolución de Cuba, Nueva York, 1871 y Como acabó la dominación de España en América, París, Garnier Hermanos, 1908.

o8 Juan Arnao, Páginas para la historia de la isla de Cuba, Habana, Imprenta "La Nueva", 1900. Publicada originalmente en 1877 es, más que una historia propiamente dicha, una memoria personal sobre la lucha por la independencia desde 1818 a 1868.

69 Néstor Ponce de León no publica más allá del prólogo y el capítulo I. "Historia de la Isla de Cuba" (publicada originalmente en 1888) en Revista de la Biblioteca Nacional, 1911, año III, t. V, núm. 1-6, p. 97-106.

${ }_{70}$ Manuel Sanguily, patriota, hombre de vasta cultura e historiador critico, escribió también un sinnúmero de artículos relacionados con la independencia de Cuba. Su obra Páginas de la historia, contiene varias monografías referidas a lo anterior. Es considerado como otro de los historiadores más valiosos de Cuba; su obra está reunida en Obras de Manuel Sanguily 3 t., La Habana, A. Dorrbecker Impresor, 1926.

${ }_{71}$ Francisco Javier Balmaceda, Los confinados de Fernando Poo, e Impresiones de un viaje a Guinea, Nueva York, Imprenta "La Revolución", 1869 y La Habana, Antonio Martin Lamy, 1899. Sobre las deportaciones de patriotas cubanos a la isla de Fernando Poo, posesión española en África.

${ }^{72}$ Francisco Javier Cisneros, Relación documentada de cinco expediciones, Nueva York, imprenta de Hallet y Breen, 1870; y La verdad histórica sobre sucesos en Cuba, Nueva York, Imprenta de Zarzamendi, 1871.

73. Fermín Valdés Dominguez, Los voluntarios de La Habana en el acontecimiento de los estudiantes de medicina, La Habana, Imprenta y papelería de Rambla y Bouza, 1909. Publicado originalmente en 1873.

${ }^{4}$ Antonio Zambrano, La república de Cuba, La Habana, Universidad de La Habana, 1969. Publicada por primera vez en 1873.

75 Ramón Roa, Convenio del Zanjón. Carta del Sr. Ramón Roa; Nueva York, Imprenta y Libreria de Néstor Ponce de León, 1878; también A pie y descalzo, de Trinidad a Cuba, 1870-1871 (Recuerdos de campaña), La Habana, Establecimiento tipográfico, 1890; ambos fueron reunidos en Con la pluma y el Machete, 3 vols., La Habana, publicaciones de la Academia de Historia de Cuba, 1950.

${ }^{76}$ Manuel de la Cruz, Episodios de la Revolución Cubana, La Habana, Establecimiento tipográfico, 1890. Como crítico de la historia, Manuel de la Cruz tiene un lugar destacado, véase cita núm. 6.

"Enrique Collazo, Desde Yara hasta el Zanjón; apuntaciones históricas, La Habana, Tipografia "La Lucha", 1893.

${ }_{78}$ Nicolás Heredia, Crónicas de la guerra de Cuba, relación detallada de las operaciones de la campaña, profusamente ilustradas con interesantes vistas y retratos tomados de fotografias directas, (publicado originalmente entre 1895 y 1896), La Habana, Academia de Historia de Cuba, 1957.

${ }^{79}$ Rafael María Merchán, Cuba; justificación de sus guerras de independencia, (publicada por primera vez en 1896), La Habana, Nacional, 1961.

${ }^{80}$ Fernando Figueredo, La Revolución de Yara 1868-1878, conferencias históricas, La Habana, Ediciones, 1969. Figueredo habia sido secretario de Céspedes y el texto lo constituyen una serie de 9 conferencias ofrecidas en Florida entre 1882 y 1886 y publicadas por primera vez en 1902. 
que fue uno de los historiadores críticos más fecundos e importantes de este periodo; publicó numerosos artículos y varias obras reconocidas. ${ }^{81}$ Si bien Bachiller y Morales no abraza explícitamente la causa independentista, su profunda crítica a la colonia española lo coloca en un lugar destacado junto a los patriotas cubanos.

Dentro de la corriente reformista de la historiografía cubana de la segunda mitad del siglo XIX podemos encontrar en principio a José Antonio Saco, tres veces diputado a Cortes en España, sin poder nunca asumir el cargo, convertido en principal inspirador del reformismo. Tal vez, al igual que Bachiller y Morales, la valoración de sus obras no se deriva tanto de sus posiciones políticas concretas, sino de la aportación historiográfica que hacen. ${ }^{82}$ Sin embargo, Saco es mucho más claro en sus preferencias politicas, las cuales estarán presentes en su obra: la lucha contra el despotismo español y a favor del abolicionismo y el antianexionismo a Estados Unidos.

Serán entusiastas seguidores del reformismo cubano como tal o disfrazado de autonomismo, José Silverio Jorrin, ${ }^{83}$ Eusebio Valdés Dominguez, ${ }^{84}$ Alfredo Zayas, ${ }^{85}$ Herminio C. Leyva ${ }^{86}$ y Enrique Trujillo. ${ }^{87}$

Finalmente, están los que se identifican con la posición colonial española y que dan la visión más conservadora del periodo. La mayoría de estos autores son españoles y, algunos, funcionarios de la corona que opinan o hacen crónicas sobre las guerras de independencia tomándolas como insurrecciones a las que hay que enfrentar. Entre los más representativos es posible señalar a Mariano Torriente; ${ }^{88}$ Dionisio Alcalá Galiano, ${ }^{89}$ Carlos de Sedano, ${ }^{90}$ José Ahumada y Centurión, ${ }^{91}$

81 Quizá la obra más importante de Bachiller y Morales sea la referida en la nota núm. 3, publicada originalmente entre 1860 y 1861 .

82 José Antonio Saco es autor de muchísimos artículos algunos de los cuales, los más importantes, que se refieren a Cuba están reunidos en Colección de Papeles científicos, históricos, políticos y de otros ramos sobre la isla de Cuba, ya publicados, ya inéditos, 3 vols., Paris, d'Aubusson y Kugelman, 1858-1859. Pero lo más reconocido de Saco es lo que dedica a la historia de la esclavitud en el mundo en diferentes obras.

${ }_{83}$ José Silverio Jorrin, España y Cuba, París, tipografia Lahure, 1876, reproducida en la Revista Cubana, t. I1, núm. 5, 1886, p. 433-461.

"t+ Eusebio Valdès Domínguez, Los antiguos diputades de (uha y apuntes para la historia constitucional de esta isla, La Habana, Imprenta "El Telégrafo", 1879.

${ }^{85}$ Alfredo Zayas, Cuba autonómica (estudios históricos), La Habana, Imprenta "El Retiro", 1889.

${ }^{86}$ Herminio C. Leyva, El movimiento insurreccional de 1879 en la provincia de Santiago de Cuba (La Guerra chiquita), La Habana, Imprenta y Papelería "La Universal", 1893. Leyva fue el fundador del Partido Liberal (Autonomista).

${ }^{87}$ Enrique Trujillo, A puntes históricos. Propaganda y movimientos revolucionarios cubanos en los Estados Unidos desde enero de 1880 hasta febrero de 1895, Nueva York, Tipografía “El Porvenir", 1846.

${ }^{88}$ Mariano Torriente, Bosquejo económico político de la isla de Cuba, compresiva de varios proyectos de prudentes y saludables mejoras que pueden introducirse en su gobierno y administración, Madrid, Imprenta de Don Manuel Pita, 1852.

${ }^{89}$ Dionisio Alcalá, Cuba en 1858, Madrid, Imprenta de Beltrán y Viñas, 1859.

90 Carlos de Sedano, Cuba estudios políticos, Madrid, Imprenta de Manuel G. Hernán$\mathrm{dez}, 1872$; y Cuba desde 1850 a 1873. Colección de informes, memorias, proyectos y antecedentes sobre el gobierno de la isla de Cuba, relativos al citado periodo que ha reunido por comisión del gobierno Dn. Carlos Sedano y Cruzat, exdiputado a Cortes, Madrid, Imprenta Nacionäl, 1873. Sedano es de los pocos cubanos que se identifican y escriben en consecuencia con la causa española.

${ }^{1}$ José Ahumada y Centurión, Memorias histórico-política de la isla de Cuba, redactada por orden del señor ministro de Ultramar, La Habana, Libreria e Imprenta de A. Pego, 1874. 
Justo Zaragoza, ${ }^{92}$ Vicente García Verdugo,,$^{9,3}$ Eleuterio Llofriu y Sagrera, ${ }^{94}$ Emilio A. Soulére, ${ }^{95}$ Francisco de Camps y Feliú, ${ }^{96}$ Antonio Pirala, ${ }^{97}$ Rafael Guerrero, ${ }^{98}$ Fernando Gómez, ${ }^{99}$ Tesifonte Gallego ${ }^{100}$ y Miguel Rodriguez Ferrer. ${ }^{101}$ En general pocos de estos autores tienen obras de calidad, la gran mayoría son apologías del colonialismo espanool, como era de esperarse, y tratan de afianzar las concepciones conservadoras en la isla de Cuba.

Una parte interesante que de alguna manera pertenece a la historiografia cubana del siglo XIX, son los libros escritos por norteamericanos, sea de sus viajes a Cuba, sea de sus visiones sobre la sociedad cubana. La importancia que Cuba tiene para los norteamericanos se muestra por este hecho también. En un artículo publicado en 1966 en una revista norteamericana ${ }^{102}$ se hace un listado de las obras referentes a la isla de Cuba. Sólo en el siglo XIX se registran 77 títulos distintos de otros tantos autores, de los cuales la mayoría - 31- fueron escritos entre 1890 y 1899.

\section{La historiografía de la República (1898-1959).}

La independencia de España lograda finalmente en 1898 va a dar entrada al periodo llamado de la República, que se extiende hasta la toma del poder por los revolucionarios, en 1959. Se caracterizará por las grandes transformaciones capitalistas impulsadas por la aguda penetración económica que llevan a cabo los norteamericanos desde finales del siglo X̄ıX y que llevará a la recién constituida nación cubana a una casi total supeditación al capital norteamericano.

Este proceso de dominación imperialista creará una economía organizada en función de la demanda de ciertos productos, fomentando aún más la monoproducción, expresada principalmente en el cultivo de la caña de azúcar y en algunos otros productos secundarios como el tabaco y la minería.

Pero si la economía cubana es subordinada a la norteamericana, ló-

92 Justo Zaragoza, Las insurrecciones en Cuba. Apuntes para la historia política de esta isla en el presente siglo, Madrid, Imprenta de Manuel G. Hernández, 1872-1873, 2 vols.

ys Vicente García Verdugo, Cuba contra España. Apuntes de un año para la historia de la rebelión de la isla de Cuba, que principió el 10 de octubre de 1868, Madrid, Imprenta y Librería Universal de los señores Crespo, 1869.

${ }^{94}$ Eleuterio Llofriu y Sagrera, Historia de la insurrección y guerra de la isla de Cuba, Madrid, Imprenta de la Galeria Literaria, 1870-1872, 4 vols.

${ }^{95}$ Emilio A. Soulére, Historia de las insurrecciones de Cuba 1869-1879, Barcelona, establecimiento tipográfico editorial de Juan Pons, 1879-1880, 2 vols.

96 Francisco de Camps y Feliú, Españoles e insurrectos. Recuerdos de la guerra de Cuba, La Habana, Establecimiento tipográfico de A. Alvarez y Compañia, 1890.

97 Antonio Pirala, Anales de la guerra de Cuba, Madrid, Felipe González Rojas, editor, 1895-1898, 3 vols.

98 Rafael Guerrero, Crónica de la guerra de Cuba, Barcelona, Librería Editorial de M. Maucci, 1895-1897, 5 vols.

99 Fernando Gómez, La insurrección por dentro. Apuntes para la historia, La Habana, M. Ruiz y Co., 1897.

100 Tesifonte Gallego, La insurrección cubana. Crónicas de la campaña, I, la preparación de la guerra, Madrid, Imprenta Central de los Ferrocarriles, 1897.

${ }_{101}$ Miguel Rodriguez-Ferrer, Naturaleza y civilización de la grandiosa isla de Cuba; o estudios variados y científicos, al alcance de todos, y otros históricos, estadísticos y políticos, Madrid, Carlos Bailly-Bailliere, 1887, 2 vols. Rodríguez-Ferrer hizo varias obras sobre Cuba. es un "liberal integrista" si cabe el concepto.

102 Harold Smith, "A Bibliography of American Traveller s book about Cuba published before 1900", The Americas, Washington, vol. 22, núm. 4, 1966; p. 404-412. 
gicamente los sectores dominantes cubanos también se subordinarán al semicoloniaje estadunidense. De hecho, la incipiente burguesía cubana había dejado de luchar desde 1878 para integrar un proyecto nacional y se conformaba con compartir el poder con la corona española. Por ello, el cambio de metrópoli no significará un enfrentamiento con los sectores dominantes cubanos, sino su integración y subordinación a los intereses de los nuevos dominadores.

En consecuencia el pensamiento que surge de la nación cubana a principios del siglo XX estará limitado por la situación de dependencia que llevará a justificar e incluso a enaltecer la presencia norteamericana (aun cuando existan sectores que, a partir de los años 20 , sostienen actitudes antimperialistas).

La historiografía cubana de esta fase republicana creció, por lo tanto, dentro de una situación de sumisión a este tipo de desarrollo capitalista y mantuvo con él una coherencia lógica que le permitió crecer ampliamente, dando importancia a temas e interpretaciones acordes con la ideología del bloque dominante.

Los estudios históricos de principios de la república mostraron, por un lado, "un debilitamiento del pensamiento cubano sobre los problemas del país", según Le Riverend ${ }^{103}$ y como contraparte, una búsqueda de temas más universales, menos relacionados con la realidad nacional, menos comprometidos; por el otro, una tendencia a la historia apologética, dedicada a exaltar héroes y al análisis psicologista de los personajes, con un predominio evidente del empirismo. Es decir, finalmente, esta historia estará influida por la corriente positivista que en Cuba sería llamada más adelante la "historiografía burguesa". Serían también obras que como en otras sociedades llevarian a la creación de los mitos, símbolos y estereotipos históricos.

Asimismo, salvo excepciones honorables, hay ausencia o escasez en - el manejo de las fuentes y la documentación y, en cambio, un uso recurrente, indiscriminado y acrítico de la bibliografía colonial, lo que demuestra la inexistencia de cuadros de historiadores profesionales que no se daban aún en el país, no obstante que desde 1910 se establece la Academia de Historia en la Universidad de La Habana, a pesar de la existencia y el esfuerzo para el funcionamiento del Archivo Nacional desde 1840, de la creación de la Oficina del Historiador de la Ciudad de La Habana (que ocupara el insigne Emilio Roig de Leuchsenring) en la tercera década de este siglo, y de la continuación de la obra de la Sociedad Económica de Amigos del País; todas estas instituciones al servicio del conocimiento histórico.

En lo general puede decirse que hay una enorme producción historiográfica durante este periodo, quizá como no la había habido hasta entonces, que sin embargo logra mantener la calidad sólo en ciertas manifestaciones importantes.

Destacan en las primeras décadas de independencia las obras monográficas de Vidal Morales y Morales, ${ }^{104}$ Emilio Bacardi, ${ }^{105}$ Manuel

${ }^{103}$ A cerca de la historia económica..., op. cit, p. 31.

104 Entre la numerosa obra de Vidal Morales y Morales sobresalen Iniciadores y primeros mártires de la revolución cubana, La Habana, Colección de Libros cubanos, 1931, publicado originalmente en 1901; y Hombres del 68, La Habana, Instituto de Ciencias Sociales, 1972, publicado por primera vez en 1904.

${ }^{105}$ Emilio Bacardí, Crónicas de Santiago de Cuba, Barcelona, tipografía de Carbonell y Esteva, 1908. 
Márquez Sterling, ${ }^{106}$ Evelio Rodríguez Lendian, ${ }^{107}$ Rafael Martínez Ortiz, ${ }^{108}$ Joaquin Llaverias. ${ }^{109}$

De igual manera deben citarse las investigaciones monográficas posteriores de Roque Garrigó, ${ }^{110}$ Francisco González del Valle, ${ }^{11}$ Adrián del Valle, ${ }^{112}$ Néstor Carbonell, ${ }^{113}$ Diego González, ${ }^{114}$ José M. Pérez Cabrera ${ }^{115}$ y Francisco Ponte Dominguez, ${ }^{116}$ publicadas todas por la Academia de Historia como una muestra del empuje productivo que llegó a tener tal institución.

Alrededor de los años treinta se inició la publicación, en la misma Academia de Historia de Cuba, de los documentos históricos cubanos que vinieron a llenar un vacío. ${ }^{17}$ También, desde 1943, el Archivo Nacional inició la labor de publicación de documentos fundamentales para la historia de Cuba.

Las historias generales tendrán sus representantes principales en Ramiro Guerra, ${ }^{118}$ Herminio Portell Vila, ${ }^{119}$ Emeterio S. Santovenia ${ }^{120}$ y la obra monumental conjunta de Ramiro Guerra, Emeterio S. Santovenia,

106 Manuel Márquez Sterling, Diplomacia en nuestra historia, La Habana, Instituto Cubano del Libro, 1967, originalmente publicado en 1909; también: Procesos históricos de la Enmienda Platt; 1897-1934, La Habana, El Siglo XX, 1941.

107 Evelio Rodríguez Lendian, La independencia absoluta como el ideal cubano, discurso leído en la Universidad de La Habana en 1899. Publicado en: Habana, Imprenta El Siglo XX, 1929; también: El Congreso de Panamá y la Independencia de Cuba, La Habana, Imprenta Avisador Comercial, 1911.

${ }^{108}$ Rafael Martínez Ortiz, Cuba: los primeros aijos de su independencia, Paris, "Le Livre Libre", 1929, 2 vols.

109 Quien fuera director del Archivo Nacional durante la ocupación norteamericana, en 1912 imprimiría su obra Historia de los Archivos de Cuba, La Habana, Academia de Historia.

${ }_{10}$ Roque Garrigó, Historia documentada de la conspiración de los soles y rayos de Bolivar, La Habana, Academia de Historia de Cuba, 1929, 2 vols.

111 Francisco González del Valle, La conspiración de la Escalera, La Habana, Academia de Historia de Cuba, 1925.

112 Adrián del Valle, Historia documentada de la conspiración de la Gran Legión del Aguila Negra, La Habana, Academia de Historia de Cuba, 1930.

${ }_{113}$ Néstor Carbonell, Los protomártires de la independencia de Cuba, La Habana, Academia de Ciencias de Cuba, 1926.

114 Diego González, Historia documentada de los movimientos revolucionarios de Cuba. de 1852-1867, La Habana, Academia de Ciencias de Cuba, 1838.

115 José Manuel Pérez Cabrera, La conspiración de 1824, La Habana, Academia de Historia de Cuba, 1936.

116 Francisco Ponte Dominguez, Historia de la Guerra de los Diez Años (desde la Asamblea de Guaimard hasta la substitución de Céspedes), La Habana, Academia de Historia de Cuba, 1958. El primer tomo se publicó en 1944.

${ }^{117}$ Constituirian estas ediciones del Archivo Nacional: Centon Epistolario de Domingo del Monte (1923); Actas de la Asamblea de Representantes y del Consejo de Gobierno; durante la Guerra de Independencia (1928); Historia documentada de San Cristóbal de La Habana en el siglo XVI (Irene Wright, 1930); Papeles existentes en el Archivo General de Indias relativas a Cuba y muy particularmente a La Habana (1931); Papeles de Marti (1933); Papeles de Maceo (1948); Archivo de Gonzalo de Quezada. Epistolario (1948) y el Diario del teniente coronel Eduardo Rosell y Malpica (1949).

118 Ramiro Guerra, Manual de Historia de Cuba: desde su descubrimiento hasta 1868 , La Habana, Instituto cubano del Libro, 1971; publicado originalmente en 1939. Anteriormente, Guerra había comenzado otra Historia de Cuba (1921-1925) de la cual sólo se publicaron 2 volúmenes que abarcan hasta 1607. Ramiro Guerra debe ser considerado como uno de los autores más importantes del periodo republicano (véase nota núm. 121).

119 Herminio Portell Vilá, Historia de Cuba en sus relaciones con Estados Unidos y España, La Habana, Jesús Montero, 1938, 4 vols.

${ }^{120}$ Emeterio S. Santovenia, Historia de Cuba, Habana, Trópico, 1942, 2 vols. 
José Manuel Pérez Cabrera y Juan J. Remos. ${ }^{121}$ Esfuerzos importantes que son el intento - quizá fallido- de difundir la historia cubana.

Dentro de los estudios de historias económicas se puede citar como las más importantes: Azúcar y Población en las Antillas de Ramiro Guerra, ${ }^{122}$ la de Fernando Ortiz, ${ }^{123}$ la del economista alemán exiliado en Cuba, H. E. Friedlander ${ }^{124}$ y la de Leví Marrero. ${ }^{125}$

Es necesario mencionar finalmente que existía un sector de la intelectualidad cubana de la república que mantuvo una actitud crítica y progresista; Ramiro Guerra, Fernando Ortiz, Emilio Roig de Leuchsenring, Raúl Cepero Bonilla y Elias Entralgo se convierten quizá en el pensamiento más importante del periodo. Son liberales que sin llegar más allá, mantienen una independencia respecto a las clases dominantes de la época. Al mismo tiempo se localiza, desde los años cuarenta, una veta marxista entre la intelectualidad cubana, encabezada por Sergio Aguirre, quien escribe el hasta ahora reconocido como primer estudio marxista en $1944,{ }^{126}$ así como Carlos Rafael Rodriguez ${ }^{127}$ y Blas Roca, ${ }^{128}$ quienes inician la corriente materialista dentro de' la historiografía cubana, continuada más tarde después de la revolución de 1959.

\section{La historiografía de la revolución.}

En 1959 se inicia el proceso de transformación que más tarde se definiría como socialista y que marcaría un cambio profundo en la sociedad cubana que repercute intensamente en el resto de los paises latinoamericanos. Este cambio en la estructura económica, politica y social tendrá inevitablemente que verse reflejado en la forma en que se concibe $y$ se hace la historia.

Hacer una evaluación de la historiografía cubana de la revolución desde afuera es una tarea riesgosa, no sólo por la limitación práctica que representa el acceso a la información de lo que se ha hecho y se está haciendo, sino también porque involucra, en principio, un sentimiento que muchas veces no permite la objetividad necesaria. Sin embargo, aunque estamos conscientes de que esta evaluación corresponde a los

121 Ramiro Guerra et al., Historia de la nación cubana, La Habana, Editorial Historia de la Nación Cubana, 1952; es la obra más amplia publicada hasta el presente sobre Cuba aunque su limitación es la exaltación de los logros de la república, pero sobresale la ausencia interpretativa que evidencia. La Riverend dice refiriéndose a ella: "En verdad no es una obra general con visión de conjunto sino una recolección de monografias", Acerca de la Historia..., op. cit., p. 40.

122 Ramiro Guerra, Azúcar y población en las Antillas, La Habana, Instıtuto Cubano del Libro, 1976; publicado originalmente en 1927 es la primera gran obra económica de la república. Más adelante, en 1940, publicaría Guerra otra obra económica: La industria azucarera.

123 Fernando Ortiz, Contrapunteo cubano del tabaco y el azúcar, Barcelona, Ariel, 1973. Publicada originalmente en 1940.

124 H. E. Friedlader, Historia económica de Cuba, La Habana, Jesús Montero editor, 1944.

125 Levi Marrero, Historia económica de Cuba, La Habana, Ed. "La Polilla", 1956.

126 Sergio Aguirre, "Seis actitudes de la burguesía cubana en el siglo XX", en Carlos Rafael Rodríguez, El Marxismo en la historia de Cuba, La Habana, Editorial Páginas, 1944, p. 27-47.

127 Carlos Rafael Rodríguez, Ibidem.

${ }_{128}$ Blas Roca, Los fundamentos del Socialismo en Cuba, La Habana, Ed. Päginas, 1943, p. 153. 
propios cientificos sociales cubanos, ${ }^{129}$ nos permitimos hacer un pequeño recuento sobre las alteraciones que sufre la actividad histórica a partir de la revolución.

La transformación socialista no sỏlo requirió modificar la sociedad cubana, sino obligadamente también un cambio profundo en la manera de observar a esa sociedad; precisó la aplicación de un nuevo instrumento, el materialismo histórico, con el cual disecar el pasado y el presente que se estaba forjando. Al proclamarse los principios del marxismo-leninismo como eje del pensamiento de la revolución, en lạs ciencias sociales nacionales se presentó naturalmente una ruptura con el pensamiento tradicional.

"No podemos vivir en la sociedad nueva con las viejas concepciones históricas: ésta es una frase repetida hasta el infinito" decía Moreno Franginal en 1966, y se preguntaba inmediatamente "Pero ¿Qué hemos hecho por la creación de la nueva historia, del nuevo historiador?". ${ }^{130}$ Ese fue precisamente el problema que hubo que enfrentar en el surgimiento de la nueva historiografia. Porque no se trataba evidentemente de aplicar por decreto el materialismo histórico en las ciencias sociales, sino de formar los cuadros que posibilitaran el surgimiento de esa nueva historia que no contaba con una tradición arraigada.

El primer intento en este sentido iría por el camino de la "profesionalización" de la historia, es decir de la creación de la infraestructura necesaria para la formación del nuevo historiador profesional. Si en el periodo anterior el historiador se formaba más que nada por voluntad propia y sobreponiéndose a las contingencias estruclurales de las ciencias sociales, ahora se trataba de crear las bases para la lormación de profesionistas con otras concepciones radicalmente dilerenciadas del momento previo.

El inicio de estas transformaciones en la infraestructura de las ciencias sociales se expresará desde 1961 con la creación de la Escuela de Historia en la Universidad de La Habana y del Instituto de Historia de la Academia de Ciencias de Cuba, reorganizando e incorporando a esta última algunos organismos que sobrevivian y continuaban trabajando, como el Archivo Nacional y la Oficina del Historiador de la Ciudad, o supliendo a otras que no cumplían su papel para la formación de los nuevos investigadores, como la Academia de Historia.

De igual manera la Biblioteca Nacional "José Marti" restructuró su labor en este periodo, continuando las investigaciones bibliográficas, editando algunos de los manuscritos que posee y especializando al personal para el manejo de la "Colección Cubana" tan importante para la historiografía nacional. Sin embargo, a pesar de los esfuerzos en tal sentido, todavia hasta 1970 más de la mitad de los títulos estaban sin catalogar. ${ }^{131}$

Por su parte al Archivo Nacional, institución indispensable para la investigación, creado durante la república, se abocó a la recuperación

129 A principios de octubre de 1984 se realizó, organizado por la Unión Nacional de Historiadores, un foro sobre la historiografia cubana desde 1959. Se presentaron diez trabajos sobre diferentes aspectos de la historiografía, a los cuales, desgraciadamente, no se pudo tener acceso, a excepción del presentado por Olga Portuondo, titulado "La historiografia cubana acerca del periodo 1510-1868 en XXV años de revolución". Sin embargo los trabajos serán publicados en 1985 en la Revista de la Biblioteca Nacional "José Marti".

130 M. Moreno Franginal, La historia como arma, op. cit.. p. 20.

131 Bohumil Bad'ura, op. cit., p. 9. 
de los archivos que se encontraban en distintas instituciones particulares u oficiales distribuidas por diferentes lugares, para preservarlos de la destrucción por ignorancia o mala fe de sus poseedores. Tarea importante del Archivo Nacional fue también la organización de archivos regionales históricos en Matanzas, Santiago y Camaguey. Evidentemente, aunque hubo una voluntad de organizar y clasificar el material en el Archivo Nacional y otros archivos de todo el país, todavía es una labor que no ha sido satisfecha totalmente ${ }^{132}$ y que requerirá el trabajo constante de las instituciones científicas.

Más adelante se crearian la Comisión de Estudios e Investigaciones Históricas de las Escuelas de Instrucción Revolucionaria del Partido Comunista Cubano, la Comisión de Historia de las Fuerzas Armadas Revolucionarias y la Comisión de Estudios Históricos del Movimiento Juvenil de Cuba, comisiones todas que asumen la responsabilidad de promover la investigación histórica sobre sus propias áreas de interés.

Este avance sin precedente en la historia del país se dirige a asegurar la creación de historiadores profesionales capacitados para proponer estudios alternos a los ya realizados y avanzar en la recuperación de la historia, armados con los instrumentos teóricos y metodológicos suficientes para ir conformando la nueva historia.

El intento de trazar esta nueva historia mostró su contraparte en las obras que se comienzan a editar después de 1959. La idea de rescatar a los clásicos del siglo XVIII y XIX y darlos a conocer al pueblo cubano lleva a reimprimir lo más importante de la obra de Urrutia, Valdés, Ramón de la Sagra, Guiteras, Bachiller, Humboldt, Saco, Varela y otros autores que se consideraron fundamentales. Del siglo XX se recuperó también en nuevas ediciones a aquellos autores que, por un lado, fueron considerados como lo más avanzado; tal és êl caso de Émilio Roig de Leuchsenring, Fernando Ortiz y Ramiro Guerra y las obras de los primeros marxistas cubanos: Sergio Aguirre, Carlos Rafael Rodríguez y Blas Roca.

Se iniciaría después la publicación de las obras históricas de la revolución, aunque algunos de los autores tuvieran producción del periodo anterior. Entre los consagrados como historiadores de la revolución, podemos enumerar a los siguientes: Manuel Moreno Franginal, Julio Le Riverend, Sergio Aguirre, Raúl Cepero Bonilla, Oscar Pino Santos, Jorge Ibarra, José Luciano Franco, Juan Pérez de la Riva, Hortencia Pichardo, Pedro Deschamps y Fernando Portuondo. Todos con amplísima bibliografía dentro de lo que quizá se podría calificar de primera generación de historiadores de la Revolución. ${ }^{133}$

En la evaluación que se tiene que hacer sobre los 25 años de historiografía durante la revolución, podemos mencionar los siguientes elementos:

I. No se ha dado todavía lo que podemos llamar la síntesis histórica como producto de la revolución. Aunque ya es claro esto y las instituciones de investigación trabajan en ello, no se ha logrado resolver el problema. Es todavía la historiografia de la revolución una historiografia fragmentada y parcializada, en la que las monografías sobre distintos aspectos son la realización principal.

132 El articulo del Bohumil Bad'ura, nos da una visión de la situación que guardaban los archivos hasta 1970, que aun no ha mejorado mucho, fuera del Archivo Nacional, según la conversación del autor de este articulo con historiadores cubanos.

133 Para un desglose más amplio de la historiografía de la revolución, léase la entrevista a Oscar Zaneti en este mismo número. 
II. Dentro de la producción monográfica surgieron nuevos temas que nunca se habian tratado: demografia, movimiento obrero, movimiento estudiantil, estudios politicoideológicos, etc., que obviamente no habian tenido espacio en la historiografía anterior y que se convierten ahora en tema recurrente.

III. Existe desequilibrio en los periodos trabajados. El más afortunado en cuanto al trabajo hecho es el periodo que va entre la guerra de los diez años y la cuarta década del siglo XX. En cambio el periodo colonial hasta 1868 prácticamente se ha olvidado, quizá por haberse trabajado tanto en el periodo republicano o quizá también por el trabajo documental que requiere. De igual manera, el periodo que va de 1935 a la revolución ha sido descuidado ampliamente. Es posible que este desequilibrio sea más grave para el periodo revolucionario donde no existe todavía una obra fundamental que explique y analice el proceso revolucionario antes y después de la toma del poder.

IV. Se da una evidente profesionalización de la historia que sin embargo se contrapone en la práctica al empirismo, vicio heredado del pasado. Si bien se ha luchado por la formación de cuadros universitarios que cuenten con una preparación teórica y metodológica adecuada, en cierta medida se carece aún de un trabajo de archivo adecuado en la investigación. Esto podría explicar la escasa investigación sobre el periodo colonial.

V. Al mismo tiempo, y éste puede ser uno de los principales errores de la historiografía que se inicia con la revolución, se aplicó un marxismo esquemático, fácilmente explicable por la juventud del proceso revolucionario. En la década de los años sesenta se formó toda una historiografía cuyo lenguaje materialista fue lo único que acercó esas obras al marxismo; o el contenido no correspondía a la proclamación del método que se decia aplicar, o la aplicación de este método se daba de manera mecánica. ${ }^{134}$ Comenzar los textos con citas de Marx, Engels, Lenin, Fidel o el Che acreditaba como marxistas a los autores. Acusar por principio al "imperialismo norteamericano" de todos los males que ocurren en Cuba, América Latina o el mundo, sin mayor análisis, fue otra divisa que se derivó de las concepciones mecanicistas. Afortunadamente en la década siguiente se criticarian estas concepciones (o desviaciones).

VI. Finalmente, otro aspecto que hasta el presente frena la creación historiográfica, o por lo menos la retrasa, es la ausencia de tecnología especializada aplicada a las ciencias sociales. Se trabaja artesanalmente, como en muchos otros países, sin posibilidad de agilizar los procesos productivos.

Tal es la visión que un observador externo tiene de la historia actual cubana. Los señalamientos de limitaciones no se hacen con el fin de descalificar la historiografía cubana, sino por el contrario, creemos que precisamente por la identificación que se tiene con su proceso revolucionario, es más importante señalar las limitaciones que tuvo o tiene que deshacerse lanzando alabanzas. Por otro lado, es evidente que mucho de lo señalado pierde valor ante el esfuerzo que honestamente se hace por superarlas y que, finalmente, serán los propios historiadores cubanos los encargados de resolverlas.

\footnotetext{
134 “Quizá el peligro mayor esté en el seudoimperialismo histórico que emerge y florece en los periodos de transición como una forma de oportunismo intelectual y que confunde lacilmente a la juventud". Moreno Franginal, op. ‘ia. p. 26.
} 